\title{
Welcome to Journal of Otorhinolaryngology, Hearing and Balance Medicine
}

\author{
Wei-Jia Kong ${ }^{1, *}$, Richard Salvi ${ }^{2}$ and Jochen Schacht ${ }^{3}$ \\ 1 Institute of Otorhinolaryngology, Union Hospital, Tongji Medical College, \\ Huazhong University of Science and Technology, Wuhan 430022, China \\ 2137 Cary Hall, Center for Hearing \& Deafness, University at Buffalo, Buffalo, NY 14214, USA; \\ salvi@buffalo.edu \\ 3 Department of Otolaryngology, Kresge Hearing Research Institute, University of Michigan, \\ Ann Arbor, MI 48109-5616, USA; schacht@umich.edu \\ * Correspondence: entwjkong@hust.edu.cn
}

Received: 24 February 2017; Accepted: 24 February 2017; Published: 28 February 2017

To date, much progress has been achieved in the field of Otorhinolaryngology—Head and Neck surgery, as well as hearing and balance science and medicine. A large number of new techniques and methods have also recently been established. In the basic research field of sensorineural hearing loss, significant advances include: the discovery of numerous inherited deafness-associated genes [1], progress in cochlear cell development and regeneration [2], new insights on acquired hearing loss and its prevention [3], as well as auditory central physiological and pathological mechanisms [4]. The pathogenesis of otogenic vertigo, and comprehensive individualized and procedural treatment programs are becoming well-established [5]. The diagnosis and retraining therapy for tinnitus have also seen breakthroughs [6]. Advances have been made in assessment of otitis media, and its classification and treatment [7]. A groundbreaking discovery in the pathogenesis of allergic rhinitis-allergic rhinitis and its impact on asthma (ARIA) - has redefined the classification of rhinitis and allergic rhinitis [8], and the mechanism and treatment of sinusitis [9]. In addition, there have been fruitful results in research related to the pathogenesis and treatment of obstructive sleep apnea-hypopnea syndrome (OSAHS) [10], the pathogenesis of head and neck neoplasms, and molecular epidemiology [11], as well as their comprehensive treatment.

In order to provide a forum for this broad clinical and scientific field, we have established a multidisciplinary publication, the Journal of Otorhinolaryngology, Hearing and Balance Medicine (OHBM). We aim to publish original research articles (including basic research, translational research as well as clinical studies), review articles and case reports in all areas of Otorhinolaryngology-Head and Neck medicine including hearing and balance science, otology, neurotology, rhinology, laryngology, head and neck surgery, bronchoesophagology, maxillofacial and plastic surgery, audiology and speech science and medicine, oncologic medicine and pediatric otorhinolaryngology, but also the quickly developing fields of allergology, skull base surgery, and sleep medicine.

We are very proud to welcome our founding Editorial Board Members, a group of more than 50 established academics and clinicians from all over the world. On behalf of the Editorial Board, we invite you to submit your manuscripts to and suggestions for OHBM and other interesting ideas for this discipline. Please see the Appendix A for the current Editorial Board leadership of the Journal of Otorhinolaryngology, Hearing and Balance Medicine (OHBM). 


\section{Appendix}

\section{Editor-in-Chief}

Wei-Jia Kong, Department of Otorhinolaryngology, Union Hospital, Tongji Medical College, Huazhong University of Science and Technology, Jiefang Avenue1277, Wuhan 430022, China

\section{Associate Editors}

Richard Salvi, 137 Cary Hall, Center for Hearing \& Deafness, University at Buffalo, Buffalo, NY 14214, USA

Jochen Schacht, Department of Otolaryngology, Kresge Hearing Research Institute, University of Michigan, Ann Arbor, MI 48109-5616, USA

\section{Editorial Broad of OHBM}

Friedrich Bootz, Department of Otorhinolaryngology/Head and Neck Surgery, University Hospital of Bonn, Bonn, Germany

Itzhak Brook, Department of Pediatrics, School of Medicine, Georgetown University, 4431 Albemarle Street, NW, Washington, DC, 20016, USA

Renjie Chai, Institute of Life Sciences, Southeast University, Nanjing 210096, China

Zheng-Yi Chen, Department of Otolaryngology, Harvard Medical School, 243 Charles St, Boston, MA 02114, USA

Ping Helen Chen, Department of Cell Biology, Emory University, 615 Michael St, 1941/001/1AF, Atlanta, GA 30322, USA

Fangyi Chen, Department of Biomedical Engineering, South University of Science and Technology of China, Shenzhen 518055, China

Lin Chen, Auditory Research Laboratory, School of Life Sciences, University of Science and Technology of China, Hefei 230027, China

Seong Ho Cho, Division of Allergy, Department of Internal Medicine, Morsani College of Medicine, University of South Florida, Tampa, FL 33620, USA

Jacquelynne P. Corey, Department of Surgery, Section of Otolaryngology-Head and Neck Surgery, University of Chicago Medical Center, Chicago, IL 60637, USA

Vincent Cousins, ENT-Otoneurology Unit, The Alfred Hospital, Melbourne, Australia Department of Surgery, Monash University, Melbourne 3800, Australia

Pu Dai, Department of Otolaryngology, PLA General Hospital, Beijing 100853, China

Dalian Ding, Center for Hearing and Deafness, Department of Communicative Disorders and Sciences, State University of New York at Buffalo, 137 Cary Hall, 3435 Main Street, Buffalo, NY 14214, USA

Maoli Duan, Department of Clinical Science, Technology and Intervention, Karolinska Hospital, Karolinska Institutet, Stockholm 14183, Sweden

Ana Belén Elgoyhen, Departamento de Farmacología Facultad de Medicina, Universidad de Buenos Aires and Instituto de Investigaciones en Ingeniería Genética y Biología Molecular, Dr. Héctor N. Torres (INGEBI), Consejo Nacional de Investigaciones Científicas y Técnicas (CONICET) Buenos Aires 1417, Argentina 
Jiangang Gao, School of Life Science, Shandong University, 27 Shanda Nanlu, Jinan 50100, China

Zhiqiang Gao, Department of Otolaryngology, Peking Union Medical College Hospital, Chinese Academy of Medical Sciences and Peking Union Medical College, Beijing 100005, China

Christos Georgalas, Academic Medical Centre, A2-228, Meibergdreef 9, Amsterdam 1105 AZ, The Netherlands

Fang Han, Department of Pulmonary, Critical Care Medicine, Peking University People's Hospital, Beijing 100044, China

Philip H.S. Jen, Division of Biological Sciences, University of Missouri, Columbia, MO 65211, USA

Jack J. Jiang, Department of Surgery, Division of Otolaryngology, Head and Neck Surgery, School of Medicine and Public Health, University of Wisconsin-Madison, Madison, WI 53706, USA

Herman Kingma, Department of Oto-Rhino-Laryngology and Head and Neck Surgery, Maastricht University Medical Centre, Maastricht 6200 MD, the Netherlands

Ja-Won Koo, Department of Otorhinolaryngology-Head \& Neck Surgery, Seoul National University Bundang Hospital, 300 Gumi-dong, Bundang-gu, Seongnam, Gyeonggi-do 463-707, Korea

Guojun Li, The University of Texas MD Anderson Cancer Center, 1515 Holcombe Boulevard, Houston, TX 77030, USA

De-Pei Li, Department of Critical Care, The University of Texas, M.D. Anderson Cancer Center, 1515 Holcombe Boulevard, Houston, TX 77030, USA

Xiaoming Li, Department of Otolaryngology Head Neck Surgery, Bethune International Peace Hospital, Shijiazhuang 050081, China

Huawei Li, Department of Otolaryngology, Affiliated Eye and ENT Hospital, Fudan University, Shanghai 200031, China

Xi Erick Lin, Dept of Otolaryngology-Head \& Neck Surgery, Emory University School of Medicine, 615 Michael Street, Whitehead Bldg \#543, Atlanta, GA 30322, USA

Zheng Liu, Department of Otolaryngology Head and Neck Surgery, Tongji Hospital, Tongji Medical College, Huazhong University of Science and Technology, Wuhan 430074, China

Duan Ma, Key Lab of Metabolism and Molecular Medicine, Ministry of Education, School of Basic Medical Sciences, Fudan University, Shanghai 200433, China

Claudina A. Pérez Novo, Upper Airways Research Laboratory, Department of Ear-, Nose-, and Throat diseases, Ghent University Hospital, Medical Research Building (MRB, groundfloor), De Pintelaan 185 9000 Ghent, Belgium

Carl Philpott, Norwich Medical School, University of East Anglia, Norwich Research Park, Norwich, Norfolk, NR4 7TJ, UK

Jianhua Qiu, Department of Otolaryngology-Head and Neck Surgery, Xijing Hospital, Fourth Military Medical University, Xi'an 710032, China

Helge Rask-Andersen, Department of Surgical Sciences, Head and Neck Surgery, Section of Otolaryngology, Uppsala University Hospital, Uppsala, Sweden

Tianying Ren, Department of Otorhinolaryngology, Oregon Health and Science University, Portland, OR 97239, USA

Anneliese Schrott-Fischer, Department of Otolaryngolgy, Medical University Innsbruck, Anichstr. 35, 6020 Innsbruck, Austria 
Michael Setzen, Department of Otolaryngology, School of Medicine, New York University, New York, NY 10276, USA

Su-hua Sha, Department of Pathology \& Laboratory Medicine, Medical University of South Carolina, 39 Sabin Street, Charleston, SC 29414, USA

Frank Wallner, Department of Otolaryngology, University Heidelberg, Heidelberg 69120, Germany

Lei Wang, Institutes of Biomedical Science, Fudan University, 138 Yixueyuan Road, Shanghai 200032, China

Qiuju Wang, Chinese PLA Institute of Otolaryngology, Chinese PLA General Hospital, Medical School of Chinese PLA, 28 Fuxing Road, Beijing 100853, China

Hao Wu, Department of Otolaryngology Head and Neck Surgery, 9th People's Hospital, School of Medicine, Shanghai Jiao Tong University, Shanghai 200092, China

Zhigang Xu, School of Life Science, Shandong University, Jinan, Shandong 250100, China

Jingying Ye, Department of otolaryngology-head neck surgery, Beijing Tsinghua Changgung Hospital, Tsinghua University, No.168 Litang Road, Dongxiaokou Town, Changping District, Beijing, China

Shankai Yin, Department of Otorhinolaryngology, Shanghai Jiao Tong University Affiliated Sixth People's Hospital, Shanghai 201306, China

Huijun Yuan, Medical Genetics Center, Southwest Hospital, Third Military Medical University, Chongqing 400038, China

Nan Zhang, Ghent University Hospital, ENT Department, Upper Airways Research Laboratory, Medical Research Building (MRB, groundfloor) De Pintelaan 185, 9000 Ghent, Belgium

Luo Zhang, Beijing Institute of Otolaryngology, Beijing 100005, China

Xiaowen Zhang, State Key Laboratory of Respiratory Disease, Department of Otolaryngology-Head and Neck Surgery, Laboratory of ENT-HNS Disease, First Affiliated Hospital, Guangzhou Medical University, Guangzhou, China

Hongliang Zheng, Department of Otorhinolaryngology-Head and Neck Surgery, Changhai Hospital, Second Military Medical University, Shanghai 200433, China

Qingyin Zheng, Department of Otolaryngology-Head and Neck Surgery, School of Medicine, Case Western Reserve University, Cleveland, OH 44106, USA

Xiaoming Zhou, Key Laboratory of Brain Functional Genomics of Ministry of Education, East China Normal University, Shanghai 200062, China

Jing Zou, Department of Otolaryngology-Head \& Neck Surgery, Changhai Hospital, Second Military Medical University, Shanghai 200433, China

\section{References}

1. Shearer, A.E.; DeLuca, A.P.; Hildebrand, M.S.; Taylor, K.R.; Gurrola, J, II; Scherer, S.; Scheetz, T.E.; Smith, R.J. Comprehensive genetic testing for hereditary hearing loss using massively parallel sequencing. Proc. Natl. Acad. Sci. USA 2010, 107, 21104-21109. [CrossRef] [PubMed]

2. Smith, M.E.; Groves, A.K.; Coffin, A.B. Editorial: Sensory Hair Cell Death and Regeneration. Front. Cell. Neurosci. 2016, 10, 208. [CrossRef] [PubMed]

3. Yang, C.H.; Schrepfer, T.; Schacht, J. Age-related hearing impairment and the triad of acquired hearing loss. Front Cell Neurosci. 2015, 9, 276. [CrossRef] [PubMed]

4. Guinan, J.J., Jr;; Salt, A.; Cheatham, M.A. Progress in cochlear physiology after Békésy. Hear Res. 2012, 293, 12-20. [CrossRef] [PubMed] 
5. Patel, M.; Agarwal, K.; Arshad, Q.; Hariri, M.; Rea, P.; Seemungal, B.M.; Golding, J.F.; Harcourt, J.P.; Bronstein, A.M. Intratympanic methylprednisolone versus gentamicin in patients with unilateral Ménière's disease: A randomised, double-blind, comparative effectiveness trial. Lancet 2016, 388, 2753-2762. [CrossRef]

6. Henry, J.A.; Roberts, L.E.; Caspary, D.M.; Theodoroff, S.M.; Salvi, R.J. Underlying mechanisms of tinnitus: Review and clinical implications. J. Am. Acad. Audiol. 2014, 25, 5-22. [CrossRef] [PubMed]

7. Schilder, A.G.; Chonmaitree, T.; Cripps, A.W.; Rosenfeld, R.M.; Casselbrant, M.L.; Haggard, M.P.; Venekamp, R.P. Otitis media. Nat. Rev. Dis. Primers 2016, 2, 16063. [CrossRef] [PubMed]

8. Bousquet, J.; Schuenemann, H.J.; Samolinski, B.; Demoly, P.; Baena-Cagnani, C.E.; Bachert, C.; Bonini, S.; Boulet, L.P.; Bousquet, P.J.; Brozek, J.L.; et al. Allergic Rhinitis and its Impact on Asthma (ARIA): Achievements in 10 years and future needs. J. Allergy Clin. Immunol. 2012, 130, 1049-1062. [CrossRef] [PubMed]

9. Bachert, C.; Gevaert, E. Advances in rhinitis and rhinosinusitis in 2015. J. Allergy Clin. Immunol. 2016, 138, 1277-1283. [CrossRef] [PubMed]

10. Jonas, D.E.; Amick, H.R.; Feltner, C.; Weber, R.P.; Arvanitis, M.; Stine, A.; Lux, L.; Harris, R.P. Screening for Obstructive Sleep Apnea in Adults: Evidence Report and Systematic Review for the US Preventive Services Task Force. JAMA 2017, 317, 415-433. [CrossRef] [PubMed]

11. Cohen, E.E.; LaMonte, S.J.; Erb, N.L.; Beckman, K.L.; Sadeghi, N.; Hutcheson, K.A.; Stubblefield, M.D.; Abbott, D.M.; Fisher, P.S.; Stein, K.D.; et al. American Cancer Society Head and Neck Cancer Survivorship Care Guideline. CA Cancer J. Clin. 2016, 66, 203-239. [CrossRef] [PubMed]

(C) 2017 by the authors. Licensee MDPI, Basel, Switzerland. This article is an open access article distributed under the terms and conditions of the Creative Commons Attribution (CC BY) license (http:/ / creativecommons.org/licenses/by/4.0/). 